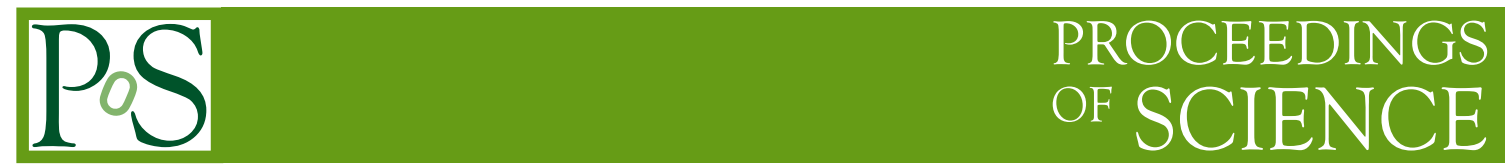

\title{
Heavy hadron spectroscopy in a Salpeter model with AdS/QCD inspired potential
}

\section{Floriana Giannuzzi*}

University of Bari and INFN

E-mail: floriana.giannuzzi@ba.infn.it

\begin{abstract}
We compute charmonium and bottomonium decay widths in two photons or two leptons and the masses of baryons comprising two heavy quarks, solving the Salpeter equation. Baryons are considered as bound states of a quark and a diquark. The interaction energy of a quark-antiquark pair comes from the AdS/QCD correspondence.
\end{abstract}

European Physical Society Europhysics Conference on High Energy Physics

July 16-22, 2009

Krakow, Poland

${ }^{*}$ Speaker. 
The AdS/QCD correspondence is a new approach for studying the non-perturbative regime of QCD. Within the bottom-up approach [1] many quantities have been computed, like, i.e., the spectrum and decay constants of light mesons and glueballs, meson form factors, QCD constants $[1,2]$, with satisfactory agreement with experimental data or predictions of other models. Another remarkable result of the AdS/QCD correspondence is the computation of the quark-antiquark potential, namely the static energy of interaction of a quark and an antiquark [3]. In the five dimensional space with metric

$$
d s^{2}=\frac{R^{2}}{z^{2}} \mathrm{e}^{c z^{2} / 2}\left(d t^{2}+d \bar{x}^{2}+d z^{2}\right),
$$

the $Q \bar{Q}$ potential is proportional to the area of the worldsheet formed by the evolution in the Euclidean time of the string connecting the quark and the antiquark, as shown in Fig. 1. The result, shown in Fig. 2, is given in a parametric form, in which the energy and the distance between the quark and the antiquark are functions of a parameter $\lambda(\lambda \in[0,2[)$ :

$$
\left\{\begin{array}{l}
V_{A d S / Q C D}(\lambda)=\frac{g}{\pi} \sqrt{\frac{c}{\lambda}}\left(-1+\int_{0}^{1} d v v^{-2}\left[\mathrm{e}^{\lambda v^{2} / 2}\left(1-v^{4} \mathrm{e}^{\lambda\left(1-v^{2}\right)}\right)^{-1 / 2}-1\right]\right) \\
r(\lambda)=2 \sqrt{\frac{\lambda}{c}} \int_{0}^{1} d v v^{2} \mathrm{e}^{\lambda\left(1-v^{2}\right) / 2}\left(1-v^{4} \mathrm{e}^{\lambda\left(1-v^{2}\right)}\right)^{-1 / 2}
\end{array} .\right.
$$

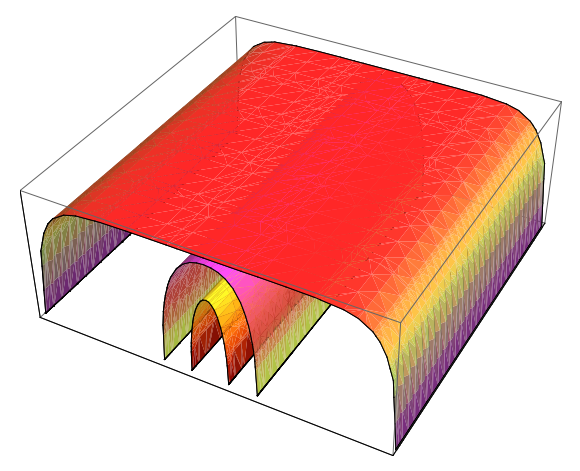

Figure 1: Worldsheet of a string connecting a static quark-antiquark pair.

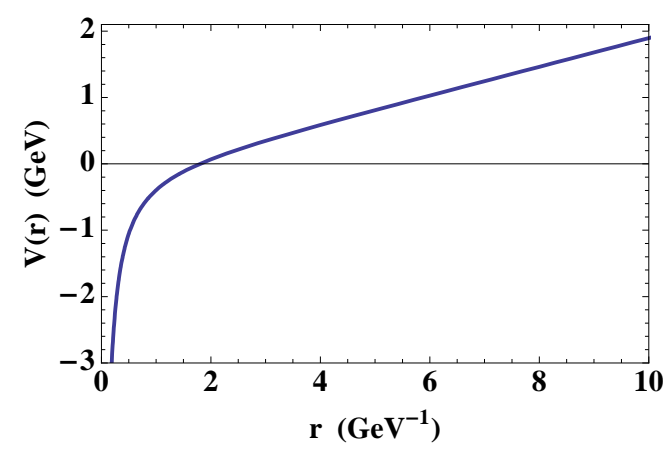

Figure 2: Static energy of a quark-antiquark pair, from Eq. (2).

In [4] the potential (2) is introduced in a potential model with relativistic kinematics to compute spectra of hadrons with at least one heavy quark. Meson masses can be obtained as eigenvalues of the Salpeter equation:

$$
\left(\sqrt{m_{1}^{2}-\nabla^{2}}+\sqrt{m_{2}^{2}-\nabla^{2}}+V(r)\right) \psi(\mathbf{r})=M \psi(\mathbf{r})
$$

where $m_{1,2}$ are the constituent masses of the quark and the antiquark, $\psi(\mathbf{r})$ and $M$ are the wave function and the mass of the meson, respectively. We have also considered the spin contribution for the potential, which, in the one gluon exchange approximation, can be written as

$$
V_{\text {spin }}(r)=A_{Q} \frac{\tilde{\delta}(r)}{m_{1} m_{2}} \mathbf{S}_{\mathbf{1}} \cdot \mathbf{S}_{\mathbf{2}} \quad \text { with } \quad \tilde{\delta}(r)=\left(\frac{\sigma}{\sqrt{\pi}}\right)^{3} e^{-\sigma^{2} r^{2}},
$$

with $A_{Q}$ and $\sigma$ parameters; since $A_{Q}$, in the one gluon exchange approximation, is proportional to the running coupling constant of QCD, it gets two different values in case of hadrons containing a 
charm or a bottom quark. So, the full interaction potential in Eq. (3) reads:

$$
V(r)=V_{A d S / Q C D}(r)+V_{\text {spin }}(r)+V_{0}
$$

with $V_{0}$ a constant term. Moreover, a cutoff should be introduced in the potential in order to avoid a divergence of the wave function, such that

$$
V(r)=V\left(r_{M}\right) \quad \text { if } \quad r<r_{M}=\frac{4 \pi \Lambda}{3 M}
$$

if $m_{1}=m_{2}$, we use $\Lambda=1$ [5].

Here, the decay constants of charmonium and bottomonium mesons and the masses of baryons comprising two heavy quarks are computed, using the parameters in [6], where the experimental data of meson spectrum [7] have been used to fit the parameters. The numerical solutions are found using the Multhopp method [8].

The following processes have been considered:

$$
\eta_{c, b}(n S) \longrightarrow \gamma \gamma \quad \psi, \Upsilon(n S) \longrightarrow l^{+} l^{-},
$$

which can be described by the effective Lagrangians [9]:

$$
\begin{aligned}
& \mathscr{L}_{e f f}^{\gamma \gamma}=-i c_{1}\left(\bar{q} \gamma^{\sigma} \gamma^{5} q\right) \varepsilon_{\mu v \rho \sigma} F^{\mu v} A^{\rho} \\
& \mathscr{L}_{e f f}^{\ell \bar{\ell}}=-c_{2}\left(\bar{q} \gamma^{\mu} q\right)\left(\ell \gamma_{\mu} \bar{\ell}\right)
\end{aligned}
$$

where

$$
c_{1}=\frac{Q^{2} 4 \pi \alpha_{e m}}{\left(M^{2}+E_{b} M\right)} \quad c_{2}=\frac{Q 4 \pi \alpha_{e m}}{M^{2}} .
$$

So, the decay widths are computed using:

$$
\Gamma_{\gamma \gamma}=\frac{4 \pi Q^{4} \alpha_{e m}^{2} M^{3} f_{P}^{2}}{\left(M^{2}+E_{b} M\right)^{2}} \quad \Gamma_{\ell^{+} \ell^{-}}=\frac{4 \pi Q^{2} \alpha_{e m}^{2} f_{V}^{2}}{3 M},
$$

where $Q$ is the electric charge (in units of $e$ ) of the constituent quark, $E_{b}=2 m-M$ is the binding energy and $f_{P, V}$ are the decay constants of the pseudoscalar and vector mesons, respectively [6]. The results are shown in Table 1, with comparisons with predictions of other models or experimental data.

The experimental branching ratio for $\eta_{c c} \rightarrow \gamma \gamma$ is $(1.8+0.6-0.5) \times 10^{-4}$ [7], from which $\Gamma_{\gamma \gamma} \sim 4.9 \mathrm{KeV}$, being very close to our prediction. The average evaluated in [7] from experimental data is $\Gamma_{\gamma \gamma} \sim(7.2 \pm 0.7 \pm 2.0) \mathrm{KeV}$ which is again compatible with our result within the error. In the case of $\eta_{c}^{\prime}$, there is an experimental observation by the Cleo Collaboration [10], measuring $(1.3 \pm 0.6) \mathrm{KeV}$; this value is smaller, or marginally comparable, with our determination. For vector mesons, there is an overall agreement with experimental results, excluding a discrepancy in the $\Upsilon(3 S)$ that could be attributed to a possible $D$-wave component in this meson.

This two-body problem can be extended to baryons and tetraquarks, considering them as bound states of a quark and a diquark and a diquark and an antidiquark, respectively. The group theory helps us in this goal, since it predicts that in the one gluon exchange approximation a bound state of two quarks, the diquark, can form in the $\overline{3}$ representation and so, from the point of view of group 
Table 1: Decay widths (in $\mathrm{KeV}$ ) of pseudoscalar states in two photons and vector states in two leptons. The values found in this paper ( $2^{\text {nd }}$ and $8^{\text {th }}$ columns) are compared with predictions of other models, in the first case, and experimental data, in the second case.

\begin{tabular}{|c|c|c|c|c|c||c|c|c|}
\hline Particle & $\Gamma_{\gamma \gamma}$ & {$[9]$} & {$[11]$} & {$[12]$} & {$[13]$} & Particle & $\Gamma_{l^{+} l^{-}}$ & Exp. [7] \\
\hline$\eta_{c}$ & 4.252 & 7.46 & 7.18 & $7.14 \pm 0.95$ & 5.5 & $J / \psi$ & 4.080 & $5.55 \pm 0.14 \pm 0.02$ \\
\hline$\eta_{c}^{\prime}$ & 3.306 & 4.1 & 1.71 & $4.44 \pm 0.48$ & 1.8 & $\psi^{\prime \prime}$ & 0.836 & $0.86 \pm 0.07$ \\
\hline$\eta_{c}^{\prime \prime}$ & 1.992 & & 1.21 & & & $\psi^{\prime \prime}$ & 0.836 & $0.86 \pm 0.07$ \\
\hline \hline$\eta_{b}$ & 0.313 & 0.560 & 0.230 & $0.384 \pm 0.047$ & 0.350 & $\Upsilon$ & 1.237 & $1.340 \pm 0.018$ \\
\hline$\eta_{b}^{\prime}$ & 0.151 & 0.269 & 0.070 & $0.191 \pm 0.025$ & 0.150 & $\Upsilon(2 S)$ & 0.581 & $0.612 \pm 0.011$ \\
\hline$\eta_{b}^{\prime \prime}$ & 0.092 & 0.208 & 0.040 & & 0.100 & $\Upsilon(3 S)$ & 0.270 & $0.443 \pm 0.008$ \\
\hline & & & & & & $\Upsilon(4 S)$ & 0.212 & $0.272 \pm 0.029$ \\
\hline
\end{tabular}

theory, the interaction between a quark and a diquark or between a diquark and an antidiquark are the same as the one between a quark and an antiquark. Meson and tetraquark masses have been computed in [4]; here we compute the spectra of baryons comprising two heavy quarks, using the same parameters as the previous calculation. In this sector, there is only one experimental result, but they could be observed by the forthcoming experiments. In order to apply to baryons the same model used for mesons, we consider the baryon a bound state of a quark and a diquark, an assumption that should properly work for doubly heavy baryons. The procedure again consists in solving the Salpeter equation (3), with $m_{1,2}$ the masses of the quark and the diquark [14]. However we know that diquarks are object with a finite size, so the potential used for baryons is a convolution of the $Q \bar{Q}$ potential (5) with the wavefunction of the diquark :

$$
\tilde{V}(R)=\frac{1}{N} \int d \mathbf{r}\left|\psi_{d}(\mathbf{r})\right|^{2} V(|\mathbf{R}+\mathbf{r}|)
$$

with $N$ a normalization factor. The masses obtained for baryons comprising two charm and bottom quarks are reported in Table 2.

Table 2: Masses $(\mathrm{GeV})$ of baryons comprising a diquark $\{c c\}_{1 S}$ or $\{b b\}_{1 S}$ and a light quark.

\begin{tabular}{|c|c|c|c|c||c|c|c|c|c|}
\hline Particle & State & $J^{P}$ & q-d content & Results & Particle & State & $J^{P}$ & q-d content & Results \\
\hline$\Xi_{c c}$ & $1 S$ & $\frac{1}{2}^{+}$ & $q\{c c\}_{1 S}$ & 3.547 & $\Xi_{b b}$ & $1 S$ & $\frac{1}{2}^{+}$ & $q\{b b\}_{1 S}$ & 10.185 \\
& $2 S$ & & & 4.183 & & $2 S$ & & & 10.751 \\
& $3 S$ & & & 4.640 & & $3 S$ & & & 11.170 \\
\hline \multirow{2}{*}{$\Xi_{c c}^{*}$} & $1 S$ & $\frac{3}{2}^{+}$ & $q\{c c\}_{1 S}$ & 3.719 & $\Xi_{b b}^{*}$ & $1 S$ & $\frac{3}{2}^{+}$ & $q\{b b\}_{1 S}$ & 10.216 \\
& $2 S$ & & & 4.282 & & $2 S$ & & & 10.770 \\
& $3 S$ & & & 4.719 & & $3 S$ & & & 11.184 \\
\hline$\Omega_{c c}$ & $1 S$ & $\frac{1}{2}^{+}$ & $\multirow{2}{*}{c c}_{1 S}$ & 3.648 & $\Omega_{b b}$ & $1 S$ & $\frac{1}{2}^{+}$ & $s\{b b\}_{1 S}$ & 10.271 \\
& $2 S$ & & & 4.268 & & $2 S$ & & & 10.830 \\
& $3 S$ & & & 4.714 & & $3 S$ & & & 11.240 \\
\hline \multirow{2}{*}{$\Omega_{c c}^{*}$} & $1 S$ & $\frac{3}{2}^{+}$ & \multirow{2}{*}{$s\{c c\}_{1 S}$} & 3.770 & $\Omega_{b b}^{*}$ & $1 S$ & $\frac{3}{2}^{+}$ & \multirow{2}{*}{$s\{b b\}_{1 S}$} & 10.289 \\
& $2 S$ & & & 4.334 & & $2 S$ & & & 10.839 \\
& $3 S$ & & & 4.766 & & $3 S$ & & & 11.247 \\
\hline
\end{tabular}


The only experimental value is the mass of $\Xi_{c c}^{+}$measured by the Selex Collaboration [15]:

$$
M_{\Xi_{c c}}=3518.9 \pm 0.9 \mathrm{MeV}
$$

the value we have obtained is comparable with the experimental one, within the approximations involved in our model and according to the agreement of our outcomes with experimental data in the meson sector.

\section{Acknowledgments}

I would like to thank M.V. Carlucci, M. Pellicoro and S. Stramaglia for collaboration on developing the numerical method used here and P. Colangelo and S. Nicotri for suggestions. This work was supported in part by the EU Contract No. MRTN-CT-2006-035482, "FLAVIAnet".

\section{References}

[1] J. Erlich, E. Katz, D. T. Son and M. A. Stephanov, Phys. Rev. Lett. 95 (2005) 261602 [hep-ph/0501128];

[2] P. Colangelo, F. De Fazio, F. Giannuzzi, F. Jugeau and S. Nicotri, Phys. Rev. D 78 (2008) 055009 [0807.1054 [hep-ph] ]; C. A. Ballon Bayona, H. Boschi-Filho and N. R. F. Braga, JHEP 0803 (2008) 064 [0711.0221 [hep-th] ]; S. J. Brodsky and G. F. de Teramond, Phys. Rev. D 77 (2008) 056007 [0707.3859 [hep-ph] ]; P. Colangelo, F. De Fazio, F. Jugeau and S. Nicotri, Phys. Lett. B 652 (2007) 73 [hep-ph/ 0703316$].$

[3] O. Andreev and V. I. Zakharov, Phys. Rev. D 74 (2006) 025023 [hep-ph/ 0604204 ].

[4] M. V. Carlucci, F. Giannuzzi, G. Nardulli, M. Pellicoro and S. Stramaglia, Eur. Phys. J. C 57 (2008) $569[0711.2014$ [hep-ph]].

[5] P. Cea and G. Nardulli, Phys. Rev. D 34 (1986) 1863.

[6] F. Giannuzzi, Phys. Rev. D 78 (2008) 117501 [0810.2736 [hep-ph] ].

[7] C. Amsler et al. [Particle Data Group], Phys. Lett. B 667 (2008) 1.

[8] P. Colangelo, G. Nardulli and M. Pietroni, Phys. Rev. D 43 (1991) 3002.

[9] J. P. Lansberg and T. N. Pham, Phys. Rev. D 74 (2006) 034001 [hep-ph / 0603113 ]; J. P. Lansberg and T. N. Pham, Phys. Rev. D 75 (2007) 017501 [hep-ph / 0609268$].$

[10] D. M. Asner et al. [CLEO Collaboration], Phys. Rev. Lett. 92 (2004) 142001 [hep-ex/0312058].

[11] O. Lakhina and E. S. Swanson, Phys. Rev. D 74 (2006) 014012 [hep-ph / 0603164 ].

[12] C. S. Kim, T. Lee and G. L. Wang, Phys. Lett. B 606 (2005) 323 [hep-ph/ 0411075 ].

[13] D. Ebert, R. N. Faustov and V. O. Galkin, Mod. Phys. Lett. A 18 (2003) 601 [hep-ph/ 0302044 ].

[14] F. Giannuzzi, Phys. Rev. D 79 (2009) 094002 [0902 . 4624 [hep-ph] ].

[15] M. Mattson et al. [SELEX Collaboration], Phys. Rev. Lett. 89 (2002) 112001 [hep-ex/ 0208014 ]; A. Ocherashvili et al. [SELEX Collaboration], Phys. Lett. B 628 (2005) 18 [hep-ex/ 0406033 ]. 